\title{
Efficient Healthcare Assisting Cloud Storage Strategy using Fog Prioritization Logic based on Edge Devices
}

\author{
K.Makanyadevi ${ }^{a}$, S.Kandasamy ${ }^{\mathrm{b}}$, Sathishkumar V E , R.Sasikumar $^{\mathrm{d}}$ and Firos. $^{\mathrm{e}}$ \\ A \\ Assistant Professor, Department of Computer Sciecne and Engineering, \\ M.Kumarasamy College of Engineering, Karur. \\ B \\ Assistant Professor (Sr.G), Department of Computer Sciecne and Engineering, KPR Institute of Engineering and \\ Technology, Coimbatore. \\ $\mathrm{C}$ \\ Assistant Professor, Department of Computer Sciecne and Engineering, Kongu Engineering College, \\ Perundurai, Erode \\ D \\ Assistant Professor, Department of Computer Sciecne and Engineering, K.Ramakrishnan College of Engineering, Trichy \\ E \\ Assistant Professor, Department of Computer Sciecne and Engineering, Rajiv Gandhi University, Doimukh, \\ ArunachalPradesh
}

Article History: Received: 11 January 2021; Accepted: 27 February 2021; Published online: 5 April 2021

\begin{abstract}
In recent years there are many researchers conducted regarding with cloud storage benefits and its efficiency improvements, but all are raising a question regarding the effectiveness and privacy. The effectiveness of the cloud storage system purely depends on the storage capacity and responsiveness of the server, in which the size of the data is large automatically the responsiveness of the storage server goes down. To avoid this issue many researchers found a lots of solution such as multiple cloud server placements, the local cloud farm fixation and so on. But all are coming too struck with the privacy issues and the security threats are more in the case of such things. These issues need to be resolved with one powerful mechanism as well as providing a good storage mechanism without any security threats. In this paper, a healthcare application is taken into consideration and introduce a new fog based cloud storage system is designed such as Intelligent Fog based Cloud Strategy using Edge Devices (IFCSED), in which this Fog Computing process provides an efficient health data storage structure to the cloud server to maintain the high priority records without considering on regular or non-prioritized records. This proposed strategy follows Edge-based Fog assistance to identify the healthcare data priority utilizing analyzing the records, identify the priority level and classify those priority records from the data and pass that to the remote cloud server as well as keep the remaining non-prioritized records into the local fog server. The fog server data can be back up with every point of interval using data backup logics. These backup assures the data protection and the integrity on the storage medium as well as the proposed approach of IFCSED eliminates the processing delay by using time complexity estimations. The data which is coming from Internet of Things (IoT) based real-world health record will be acquired by using controllers and other related devices, which will be delivered to the Edge Devices for manipulation. In this Edge processing device accumulates the incoming health data and classifies that based on the prioritization logic. The health sensor data which is coming in regular interval with normal sensor values are considered to be the regular non-prioritized data and the health sensor data coming up with some abnormal contents such as mismatched heart rate, increased pressure level and so on are considered as a the prioritized record. The sensor assisted health data will low-priority will be moved to the fog server, which is locally maintained into the environment itself and the sensor assisted health data coming up with high priority will be moved into the remote cloud server. The proposed approach assures the time efficiency, reduction in data loss, data integrity and the storage efficiency, as well as these things, will be proved over the resulting sections with proper graphical results.
\end{abstract}

Keywords: Cloud Computing, Healthcare, Fog Processing, Edge Devices, Intelligent Fog based Cloud Strategy using Edge Devices, IFCSED, Data Prioritization

\section{Introduction}

Cloud computing utilizes the Internet services to include computation and communication facilities for a variety of industries. Delay-sensitive technologies such as intelligent healthcare and city applications, on the other hand, now demand computation over massive amounts of sensor assisted health data distributed to consolidated cloud computing environment, resulting in a drop in device output. In comparison with cloud services, the latest frameworks of fog processing units and edge devices logic offer new technologies by reallocating benefits closer to consumers and providing less delay and power efficiency. For attain better consumption and performance, better Fault tolerance as well as a greater amount of confidentiality and support, it is critical to determine the best location of facilities and benefits throughout the 3 category IoT. Fog computing has been implemented as a strong alternative to the cloud to manage the technology and communication needs of the Internet-of-Things in current history, with the introduction of the Internet of Things (IoT) [3]. The interaction and collaboration in between edge computing devices and the fog nature with internet assistance have increasingly gotten a lot of press. The sensor assisted health data prioritization based cloud enabled fog processing framework is considered in this paper, in 
which a fog provider can take advantage of cooperation among its same fog processing units and leased cloud nodes to efficiently execute users' large-scale offloading operations. [11] The computer, fog, and cloud layers are the three key layers in a fog cloud infrastructure. Virtualization is a layer among computer and cloud computing that contains a wide range of Internet of Things devices with computing, communications and other capabilities. Requests with high latency criteria should be handled at the fog layer, though some applications can be sent to the cloud, since the fog nodes are expected to be located close the edge network [5][6].

Furthermore[12], since it has central processing power, it can minimize the bandwidth consumption and costs. The acquired demands as well as information are filtered, analyzed and preprocessed by the fog layer. The assignment is programmed throughout the edge devices unless the perception layer has sufficiently resources available and also can implement it. [13]That though, unless the function can all be completed due to a lack of resources, it would be sent to the central cloud. In such a cloud fog scenario, the sensor assisted health data optimization logic is first formulated, and then a novel edge assisted fog estimation technique is designed, with the main goal of achieving a correlation between some of the computation time and the service requirements of data centers. The empirical results show that proposed models outperform several developed architectures in terms of tradeoff value. Fog computing is an emerging approach for dealing with the multitude of Web devices. Fog computing is introducedto provide assistance to the cloud further and the associated devices can generate and operate with respect to sensor assisted health related metrics. Cloud technology with fog and edge processing units motto is to manipulate the part of cloud workload locally with respect to fog nodes, in which it react on the frontend processing servers between front end IoT devices and backend cloud servers, rather than transferring all processing to backend clouds. Fog nodes can conduct connectivity operations while interference insensitive and wide scale tasks can still be effectively processed by the cloud by placing resources at the network's edge, only one or two servers from the data sources [8]. Furthermore, if the fog nodes' resources are insufficient, the cloud's expense and complexity advantages will assist the fog in serving because someone of IoT users. [15] In addition, many applications, particularly in the Internet of Things and fog data analysis, necessitate interaction and coordination in between edge and the cloud. Fog computing, in this context, is not intended to replace cloud computing, but rather to supplement it in a new computing model known as cloud fog computing, which is designed to meet the demands of users for increasingly complex applications [7][14].

In this paper, the Intelligent Fog based Cloud Strategy using Edge Devices (IFCSED) is a novel fog powered cloud computing strategy that ensures storage efficiency and robustness. Fog nodes are local tools, such as controllers, adapters, video listening devices and other appliances with networking, computing and wireless connections. A simplistic model has been that a business area will introduce multiple fog nodes on multiple shifts aim of providing Internet access and get some activated services to its clients (such as user interfaces, commercial delivery, and input collection). During peak hours, however, certain fog processing technologies are insufficient to support customers effectively. Meanwhile, the fog provider, in this case the shopping centre, will expand its facilities by spending for the cloud server's outsourced computing and storage services, which could be virtual machines leased on a pay-per-use basis from cloud providers [9]. A service manager, which is an energy management aspect and scheduler for enterprise applications requested from consumers on the fog' side, manages all map reduce programming entities such as cloud/fog. In this circumstance, a data prioritization based cloud establishments can reduce the workflow time duration but results in higher financial burden, is not the best option for fog distributors [10]. As a result, a data optimization logic is proposed in this paper to maintain a desired trade - offs between configuration processing time and cloud resource cost. In comparison to other works, the empirical outcomes demonstrate that our approach performs exceptionally well. The major contributions of this paper are summarized as follows:

(i) To develop a novel cloud storage mechanism for processing a huge sensor assisted health data in an intelligent manner without any time delay and processing accuracy lacking with proper privacy assurance.

(ii) To implement the task prioritization logic for managing the sensor assisted health data in an efficient manner on both local (fog) and remote (cloud) storage units.

(iii) To propose a new methodology with respect to Internet of Things (IoT) logic for improving the responsiveness, speed and durability of the storage unit by using the fog enabled mechanisms.

(iv) The edge computing devices are interfaced with the proposed logic to provide the higher efficiency of sensor assisted health data prioritization.

The rest of this paper describe regarding Related Study over section 2, further section of Section 3 illustrates the proposed system methodologies in detail and Section 4 illustrates the Results and Discussion portion of the paper and the final section, Section 5 illustrates the concept of Conclusion and Future Scope of the proposed paper. These all will be explained in detail over the further section summaries.

\section{Related Study}

In the year of 2020 [1], the authors "KoenTange et al., [1]" proposed a paper related to Cloud Computing data handling strategies with respect to the survey of Internet of Things, Fog Computing and Edge Processing logics. In this paper [1], the authors described such as in recent years both researchers and professionals received a huge amount of attention from the [16] IoT devices, Mobile technology, Artificial Intelligence and Edge Devices. However, it is unlikely to obtain throughout the literature a clear and clear interpretation of certain computer methodologies and about their correlations. [17][18]The above terminologies make it very complex scientists in 
this field to go and get a particular link of such philosophies. This paper addresses this imbalance, which is a useful resource for beginners. Firstly, the aspects of contemporary device methodologies get the appreciation through similar literature. Then, so every methodology is addressed and its key elements and relations in the other are well defined. [19][20] Further,this paper [1] discussed regarding Cloud services thoroughly and notesits excellent role as a sealant among Artificial intelligence, Fog data centers and Edge information technology. Finally, we are presenting briefly open problems and potential research guidelines for computing Internet of Things; Fog computing, Cloud Data Center Processing and Fog Systems.[21]

In the year of 2020 [2], the authors "Junbin-Liang et al., [2]" proposed a paper related to cloud based feedback estimations with respect to fog data maintenance logics as well as this approach [2] illustrates the concepts of sensor based trust enabled cloud service mechanisms in detail with proper specifications. In this paper [2], the authors described such as Social Cloud Sensor is a new type of Internet of Things, combined with social networking, wireless sensors, cloud services, and edge devices. The fog computing devices are distributed along the rim of cloud technology to provide convenient, open and fully functional SSC services. Fog processor architectures can method as well as process information autonomously as well as responses quite fast in SSC. The SSC sensor layer faces various kinds of visual vulnerabilities and information sharing threats like falsification, response exploitation, feedback targets but mostly complex data threats result in a lack of self assurance among SSC socioeconomic sensing devices and virtualized network infrastructure. The confidence assessment between some of the detection devices and infrastructure is indeed appropriate. However, the computation of the robustness of the socioeconomic wearable sensors in cloud computing environment will help to create a great deal of confidence overhead computing, overhead communicative delays, that also impede SSC providers wide spread application. In order to tackle this problem, a reliable confidence virtualization method is implemented consists of multi feedback as well as network virtualization fusion. The first step is to develop a new metric for the confidence of socioeconomic sensor network and to collect multisource feedback on the trust value of sensing layers to improve malware from entering feedback nodes. Second, the sensing layer authenticity response data is collected by fog computing devices, and a recommender systems confidence estimation is carried out to reduce communication delays and overhead computing. Third, a fusion algorithm is designed to add different types of feedback trust values that overcome the limitation of trusted weights in traditional trust mechanisms in illusionary measurement and contextual weighting. Theoretical analysis of the confidence computing mechanism as well as computation findings proved that it improves algorithmic performance and accuracy compared to the existing computing methodologies.

In the year of 2020 [4], the authors "Haoxin-Wang et al., [4]" proposed a paper related to define an alternate solution to the vehicular network devices by using Cloud, Edge and Fog Computing strategies. In this paper [4], the authors described such as the vehicles participate more actively in real people lives, prerequisites for cleaner and healthier driving have emerged. Wearable devices do provide technology for completing specific and attract wide-ranging attention of both government and practitioners. The above practices ensure a very well computer infrastructure that supports portfolio application's Quality-of-Service and it can help process extensive as well as large computational services by computer offloading methodologies like cloud data center, edge computing and fog processing. In addition, various cloud, edge and fog computer configurations are adequate to support various types of computer assisted systems with massively different Quality-of-Service parameters that show how important the computer design concept is. Nevertheless, much of the existing cloud/edge/fog surveys on CVs overlook computer system architecture, where I they focus only on one particular computer architecture and (ii) they fail to discuss economic advantages, proposed solutions and process needs for traditional geometric solutions. In this paper [4], an extensive survey is presented to various computer assisted cloud, edge and fog available options. This paper [4] provides an extensive study of new regulatory architecture alternative solutions predicated on cloud, edge and fog processing frameworks, in which it proposes a new cloud computing specification of edge devices and a comparative analysis of global applications numerous cloud processing literatures assistance.

\section{Methodologies}

In this paper, a novel Intelligent Fog based Cloud Strategy using Edge Devices (IFCSED) methodology is implemented with enhanced service logics. This approach associates many technologies to provide an efficient and integrated cloud storage schemes with the integration of Fog Services in tie up with Edge Device architecture. These metrics created the powerful platform for cloud environment to store the user resources in an innovative way with remote server as well as locally enabled fog server based sensor assisted health data handling schemes. The data prioritization logic is applied over the proposed system architecture to identify the priorities present into the sensor assisted health data by using Edge Services. The edge devices are placed into the health related data handling medium such as controller or Internet of Things devices, in which it accumulates the sensor assisted health data from the respective control unit and process it accordingly with respect to the prioritization scheme. The health data is considered to be non-prioritized if it has some regular periodical contents instead of having some important metrics such as mismatched heartbeat rate, abnormal blood pressure levels and relevant measures. The health data which contains the sensitive information as well as that is non-existing into the server for a specific period of time; those will be treated as a prioritized sensor assisted health data.

The sensor assisted health data which contains the prioritized metrics will be pushed into the remote cloud server and the data which contains the regular health data metrics will be maintained it into the fog server. The machine learning based data training mechanism is handled over the proposed approach to train the model for data prioritization process. The fog server details need to be maintained by the server administrator as well as the data 
need to be periodically back up for maintenance purpose. The fog server maintenance cost is comparatively low as traditional cloud servers as well as the privacy association of such fog systems are higher than the classical data maintenance scheme presented into the cloud environment. The sensor assisted health data accumulated from the end-unit edge devices are classified with respect to the data prioritization logic and move forward to the storage units accordingly. The storage unit is deviated into two forms such as locally established server unit (fog) and remotely enabled server unit (cloud). The following equation shows the fitness estimation of the proposed data prioritization logic in association with the cloud environment, in which the financial abilities of the cloud server maintenance is estimated with respect to this metric as well the data storage success ratio is estimated with respect to this procedure.

$\left[\mathrm{f}(\mathrm{x}) \rrbracket^{\wedge}(1-\mathrm{n})=\left((\mathrm{c}) \mathrm{i}^{\wedge}(1-\mathrm{n})\right) / \mathrm{SR}\right.$

Where $\mathrm{f}(\mathrm{x})$ indicates the fitness function variable and the ratio 1 to $\mathrm{n}$ indicates the number of records maintained into the server. The term c indicates the storage cost expense as well as the SR indicates the success ratio of the data storage into the server end. The following figure, Fig-1 illustrates the overall architectural view of the proposed approach in clear manner.

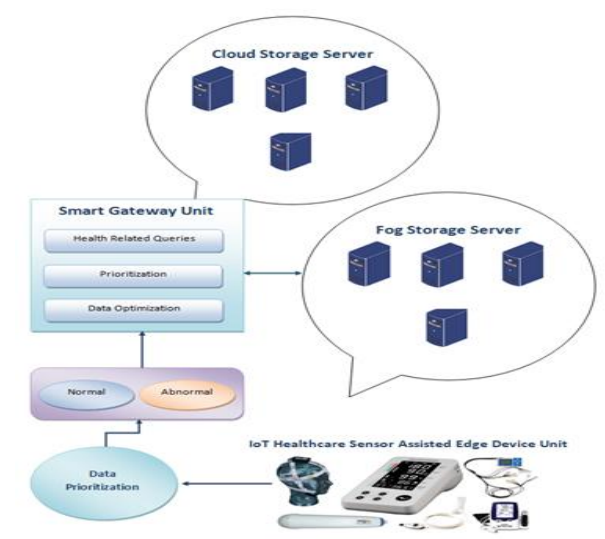

Fig.1 IFCSED Architectural Design

The following algorithm illustrates the logic of proposed approach data prioritization logic in intense manner with proper Pseudocode.

\section{Algorithm: Data Prioritization Logic}

Input:Sensor assisted health record collected from Internet of Things

Output:Classification of Priority Result with related metric features and Server Storage Ratio (Fog or Cloud).

\section{Accumulating the extracted data from the Internet of Things sensor unit with features and related threshold} metrics.

2. Analyze the priority based on the extracted sensor assisted health data attributes and test data content.

Pseudocode:

$$
\begin{aligned}
& \text { for(records.length }<0 \text {; records.count }++)\{ \\
& \qquad \text { dataset }\{i, j\}(x+1)>>\text { TestData }(i\{j\}(x+1)
\end{aligned}
$$

\section{\}}

3. Obtain the sensor-assisted health content for classification.

Pseudocode:

$$
\begin{aligned}
& \text { declare network_weights(data, thershold_value); } \\
& \text { declare task_priority; } \\
& \text { dof } \\
& \text { for(x as each_training_data,data_priority)\{ } \\
& \text { data_prediction<<neural_net_output(data_len, x); } \\
& \text { actual_data<<trained_output(x); } \\
& \text { task_priority+=random_classi_ratio(data_prediction, actual_data); } \\
& \text { \} } \\
& \text { \} }
\end{aligned}
$$




\section{return(task_priority);}

\section{Gathering the returned task priority from Step-3.}

5. Identify the sensor assisted health data priority and decide the server to store the respective data.

\section{Results and Discussion}

This paper concentrates on improved healthcare management system with respect to efficient data management principles such as avoiding data overhead, enhanced data maintenance based on prioritization logic, storage and response efficiency improvements and so on. These metrics are properly implemented by using the proposed methodology called Intelligent Fog based Cloud Strategy using Edge Devices (IFCSED). By using this approach the sensor assisted health data is prioritized and stored accordingly into the remote cloud server and the locally maintained fog server. The sensor assisted health data comes up with important metrics such as mismatched heart rate of the respective patient or blood pressure ratio changes will be considered as a high priority data and that will be stored into the remote clod server. These details will be processed accordingly to the logic mentioned in algorithm given in the methodologies section. The following figure, Fig-2 illustrates the proposed system data retrieval efficiency with respect to data collection rate of the IoT Edge device connected into the smart sensor unit of the health care system.

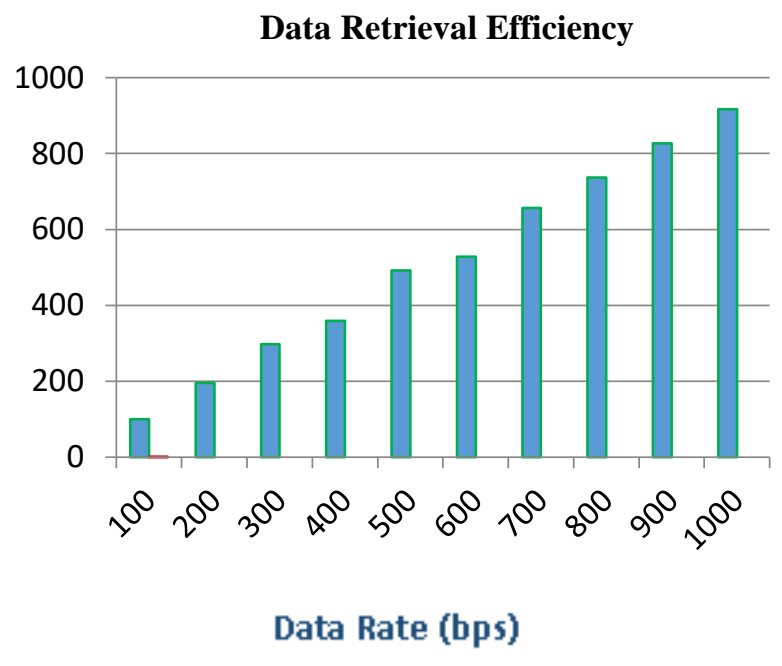

Fig.2Data Retrieval Efficiency

The following figure, Fig-3 illustrates the proposed approach cost efficiency ratio in association with the comparison of existing system classical cloud cost efficiency scenario analysis, in which the classical cloud environment health related data storage scheme provides average cost benefits to the consumers but the proposed approach cloud cost efficiency is comparatively high and that will be clearly specified into the following figure in graphical manner. 


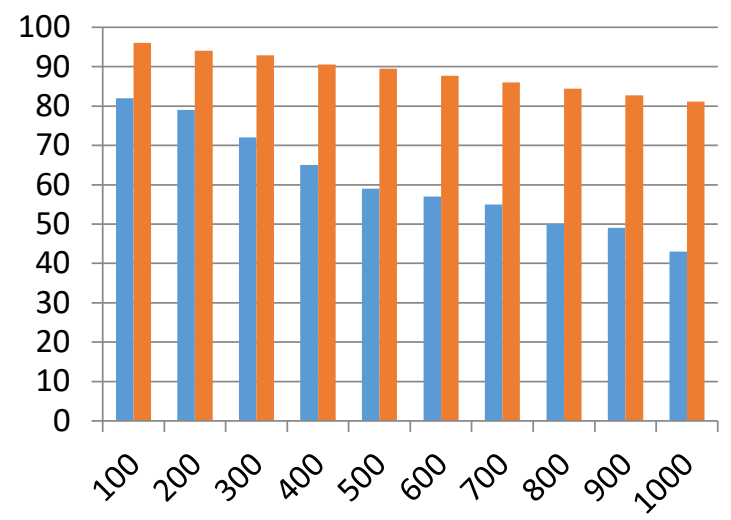

Fig.3Cost Efficiency

The following figure, Fig-4 illustrates the data loss occurred into the proposed approach of data collection over the remote server from the front end edge enabled smart sensor devices. These health related sensor details are accumulated from the real-time environment and process those details by using the Internet of Things data processing medium and the loss ratio of the proposed approach is estimated and the outcome proof is portrayed as below in graphical manner, in which it shows the comparison of classical cloud server maintenance error ratio and the proposed approach error estimation ratio.

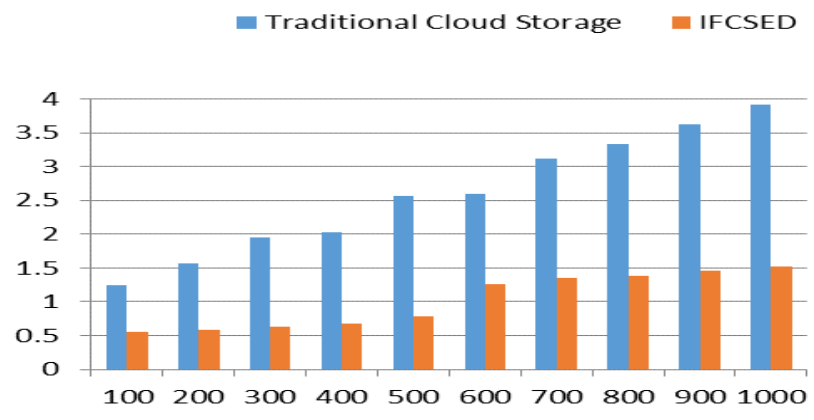

Fig.4Error Ratio

The following figure, Fig-5 illustrates the storage efficiency of the proposed approach in comparison with the classical cloud server health data maintenance scheme, in which it will be portrayed in clear manner with graphical proof, in which the $\mathrm{x}$-axis illustrates the data rate of the proposed approach in bits per second as well as the $y$-axis shows the storage efficiency in percentage.

Traditional Cloud Storage $\quad$ IFCSED

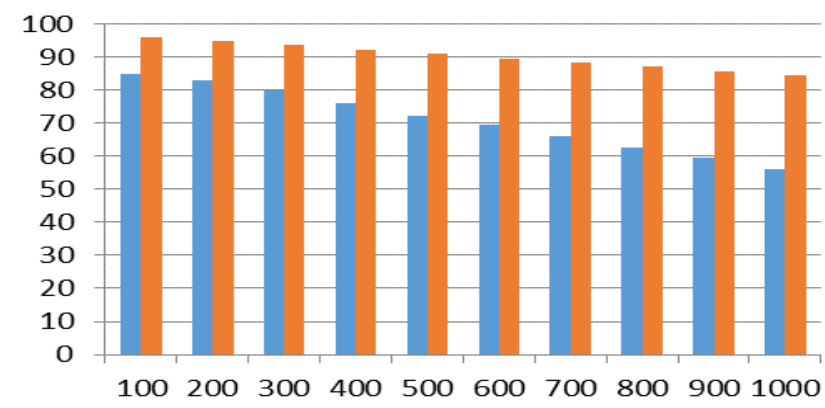

Fig.5Storage Efficiency 


\section{Conclusion and Future Work}

In this paper, a health care maintenance scheme is considered and provides efficiency to that approach by means of associating the latest technologies such as Cloud Server Handling with fog server and the edge devices. These all are integrated together to provide an efficient sensor assisted health data processing system with data prioritization logic. The proposed logic of healthcare data maintenance proves the efficiency of the implementation in several ways such as improved storage efficiency, low error rate and improved data retrieval efficiency. The specified all features are clearly proved with respect to the resulting section outcomes. The work flow and the logic of the proposed approach data prioritization logic is illustrated into the methodologies section algorithm in clear manner. The proposed approach Intelligent Fog based Cloud Strategy using Edge Devices storage efficiency is clearly illustrated into the resulting section figure, Fig-5 and the error ratio estimations are clearly illustrated with the figure, Fig-4. The cost efficiency ratio is clearly portrayed into the figure; Fig-3 over the outcome unit as well as the data retrieval efficiency of the proposed approach is clearly proved by using figure, Fig- 2 in resulting section. For all the proposed approach of Intelligent Fog based Cloud Strategy using Edge Devices (IFCSED) is more efficient and novel to provide a sufficient support to healthcare industry in proper manner.

In future, the work can further be extended by means of adding some deep machine learning technologies to provide the processing accuracy in next level as well as the processing time frequency can also be improved by means of adding such technologies over IFCSED.

\section{References}

1. Michele De Donno, Koen Tange and Nicola Dragoni, "Foundations and Evolution of Modern Computing Paradigms: Cloud, IoT, Edge, and Fog", IEEE Access, 2020.

2. Junbin Liang, Min Zhang and Victor C. M. Leung, "A Reliable Trust Computing Mechanism Based on Multisource Feedback and Fog Computing in Social Sensor Cloud",IEEE Internet of Things Journal, 2020.

3. Jingxian Liu, KeXiong, Derrick Wing Kwan Ng, Pingyi Fan, Zhangdui Zhong and Khaled Ben Letaief, "Max-Min Energy Balance in Wireless-Powered Hierarchical Fog-Cloud Computing Networks", IEEE Transactions on Wireless Communications, 2020.

4. Haoxin Wang, Tingting Liu, BaekGyu Kim, Chung-Wei Lin, Shinichi Shiraishi, Jiang Xie and Zhu Han, "Architectural Design Alternatives Based on Cloud/Edge/Fog Computing for Connected Vehicles", IEEE Communications Surveys \& Tutorials, 2020.

5. Di Wu and Nirwan Ansari, "A Cooperative Computing Strategy for Blockchain-Secured Fog Computing", IEEE Internet of Things Journal, 2020.

6. Hatem A. Alharbi, Taisir E. H. Elgorashi, and Jaafar M. H. Elmirghani, "Energy-Efficient Virtual Machines Placement Over Cloud-Fog Network Architecture", IEEE Access, 2020.

7. Huaiying Sun, Huiqun Yu and Guisheng Fan, "Contract-Based Resource Sharing for Time Effective Task Scheduling in Fog-Cloud Environment", IEEE Transactions on Network and Service Management, 2020.

8. Shudong Wang, Tianyu Zhao and Shanchen Pang, "Task Scheduling Algorithm Based on Improved Firework Algorithm in Fog Computing", IEEE Access, 2020.

9. Mohammed A. Aleisa, Abdullah Abuhussein, and Frederick T. Sheldon, "Access Control in Fog Computing: Challenges and Research Agenda", IEEE Access, 2020.

10. Carla Mouradian, Fereshteh Ebrahimnezhad, et al., "An IoT Platform-as-a-Service for NFV-Based Hybrid Cloud/Fog Systems", IEEE Internet of Things Journal, 2020.

11. R.SasiKumar \& S.DeviPriya, "Virtual Environment for Treating Anxiety Disorder using GVR Algorithm with Artificial Intelligence", International Journal of Innovative Research in Science Engineering and Technology, pp-2319-8753 Vol.8, Issue 6 June 2019.

12. R.SasiKumar, R.Ramesh, \& R.Meena, "Deep learning for Informatics using Fuzzy Logic", International Journal of Innovative Research in Science, Engineering and Technology, pp-2319-8753 Vol.8, Issue.6 June 2019.

13. R.Sasi Kumar \& S.DeviPriya,(2019), "Combining Deduplication and String Comparison for Avoiding Redundant Data with Enhanced Authentication Approach on Cloud", International Journal of Advanced Research in Computer and Communication Engineering, Issue6, Vol.8 pp-2278-1021.

14. R.Sasikumar, B.Haritha, T.Borshiya Vincy, M.Kamali \& S.De va Priya," Alumni Info-Com with Distinct Classification of Data using Support Vector Machine Algorithm", International Journal of Recent Technology and Engineering (IJRTE), 2277-3878, Volume-8 Issue-6, March 2020.

15. T.M.Nithya, J. Ramya, L. Amudha,"Scope Prediction Utilizing Support Vector Machine for Career Opportunities", International Journal of Engineering and Advanced Technology (IJEAT), ISSN: 22498958, Volume-8 Issue-5, June 2019, pp.2759-2762.

16. L. Amudha, Dr.R.PushpaLakshmi, "Scalable and Reliable Deep Learning Model to Handle Real-Time Streaming Data", International Journal of Engineering and Advanced Technology, ISSN: 2249 - 8958, Volume-9 Issue-3, February, DOI: 10.35940/ijeat.C6272.029320, 2020, Retrieval Number: C6272029320/2020 CBEIESP, pp. 3840 - 3844

17. T.M.Nithya, K.S.Guruprakash, L.Amudha. (2020). DEEP LEARNING BASED PREDICTION MODEL FOR COURSE REGISTRATION SYSTEM. International Journal of Advanced Science and Technology, 29(7s), 2178-2184 
18. Nithya, T.M., Chitra, S.. (2020). Soft computing-based semi-automated test case selection using gradientbased techniques. Soft Computing. 24. 12981-12987 (2020)

19. K.S.Guruprakash, R.Ramesh, Abinaya K, Libereta A, Lisa Evanjiline L, Madhumitha B. (2020). Optimized Workload Assigning System Using Particle Swarm Optimization. International Journal of Advanced Science and Technology, 29(7), 270

20. T.Vigneshwaran, K.S.Guruprakash, K.Thaslima Nasreen, M.Supraja,(2020), Effective Framework for real time video face recognition system, Journal of Advanced Research in Dynamical and Control system, Vol 12, Issue 6, pp680-684

21. K. S. Guruprakash, M. Jaiganesh, V. Vijey Nathan, R. Sathya. (2021). Location and Temporal based Multi-Cloud Package Selection for Enterprise. Annals of the Romanian Society for Cell Biology, 25(2), $1066-1075$. 